\title{
Postoperative Satisfaction in Patients Undergoing Fracture Neck Femur Fixation After Continuous Facia Iliaca Block (CFIB) Versus Continuous Lumbar Plexus Block (CLPB)
}

\author{
MOHAMMED A. HASSAN, M.D.; HALA E. MOHAMMED, M.D.; AMR Z. MANSOUR, M.D. and \\ MOHAMMED A. MANSOUR, M.D.
}

The Department of Anaesthesiology, Surgical ICU and Pain Management, Faculty of Medicine, Cairo University

\begin{abstract}
Background: It is thought that both CFIB and CLPB can decrease the length of hospital stay, morbidity and help early ambulation in patients with fracture neck of femur.

Aim of the Study: To compare the postoperative analgesic efficacy and opioid spare effect of continuous ultrasoundguided lumbar plexus block and fascia iliaca compartment block in patients undergoing surgery for fractured neck of femur.

Material and Methods: 40 patients with fracture neck femur were randomized intone of 2 blocks, CFIB and CLPB.

Results: CFIB was superior to CLPB in total consumed dose of morphine, sensory and motor block for 48 hours postoperatively ( $p=0.006)$, hemodynamic stability $(p<0.001)$, incidence of side effects $(p=0.04)$ and patient satisfaction $(p=0.06)$.

Conclusion: Single injection lumbar plexus block provides postoperative analgesia for up to 18 hours, with avoidance of troubles of continuous infusion, with lower VAS scores and good patient satisfaction. However, it is considered a difficult technique with higher rate of complications and failure. On the other hand, Continuous infusion fascia iliaca block gives better quality analgesia, for up to 48 hours, and high patient satisfaction.
\end{abstract}

Key Words: Continuous facia iliaca block-Continuous lumbar plexus block - Fracture neck femur anaesthesia - Postoperative pain - Patient satisfaction.

\section{Introduction}

THE fascia iliaca compartment block (FICB) is a simple, inexpensive method to induce perioperative analgesia in patients with painful conditions affecting the lower limb. Ultrasound guidance to identify the fascial planes may lead to faster onset, denser nerve blockade and an increased rate of successful blocks [1].

Correspondence to: Dr. Mohammed A. Hassan, E-Mail: Dr mohammedalihassan82@yahoo.com
Lumbar plexus block (LPB) is also referred to as the psoas compartment block as it can be achieved by local anaesthesia (LA) placement within the fascial compartment of the psoas major muscle [2].

Patients with neck femur fracture are mostly of old age and there are concerns regarding the perioperative pain, side effects of systemic medications and other complications. It is thought that both CFIB and CLPB can decrease the length of hospital stay, morbidity and help early ambulation in these patients [3]. Ultrasound guidance during induction of these two blocks, however, may increase the safety and efficacy of both techniques and higher success rates and reduced needle-related complications [4].

\section{Patients and Methods}

This prospective randomized controlled trail was carried out at Department of Anesthesiology, Intensive Care and Pain Management, Kasr ElAiny Hospital from July 2014 to July 2015, after permission from the Hospitals Ethics committee and written informed patients consent.

Forty patients, belonging to American society of Anesthesiologists physical status (ASA) I to III, between age 40 to 70 years old scheduled for fracture neck femur fixation were selected and divided into two equal groups 20 patients each (Group continuous fascia iliaca block (CFIB) and Group continuous lumbar plexus block (CLPB). Informed written consent was taken from the patients selected for the study. Each patient was visited in the ward, the evening before surgery for detailed pre-anesthesia assessment including history taking, general and local examination and routine 
preoperative labs such as CBC, liver and kidney function tests and coagulation profile.

\section{Inclusion criteria:}

- Patients American Society of Anesthesiologists status (ASA) I to III.

- Age group: 40 to 70 years; scheduled for neck femur fracture fixation surgery.

- BMI: $20-30 \mathrm{~kg} / \mathrm{m}^{2}$.

- Gender: Both sexes.

- Patient accepted technique of treatment.

- Expected operative time 2-4 hours.

\section{Exclusion criteria:}

- ASA physical status score of more than III.

- Patient with BMI of more than $30 \mathrm{~kg} / \mathrm{m}^{2}$.

- Patients refusing the procedure or uncooperative or needed GA.

- Local sepsis or infection at puncture site.

- Allergy to any of the drugs used in the study.

- Diabetic peripheral neuropathy or have a history of stroke with lesion affecting the side to surgery were excluded from the study.

- INR $>1.5$ or $<12$ hours post LMWH (many practitioners consider a posterior approach to lumbar plexus comparable to central neuroaxial blockade).

\section{Methodology in details:}

Preoperative assessment:

Routine preoperative assessment was done for every patient including:

\section{A- History:}

For general or local anesthesia and any accompanied complications, medical problem and history of drug intake.

\section{B- Examination:}

\section{General examination:}

- Vital data (pulse, blood pressure and respiratory rate).

- Clinical examination of the chest and heart.

- Examination for jaundice, cyanosis, anemia, clubbing and edema.

\section{Local examination:}

- Airway examination.

- Examination to the site of injection.

\section{C- Investigations:}

- Complete blood count.
- Coagulation profile (prothrombin time and concentration).

- Liver and kidney function test.

- Random blood sugar.

- ECG.

1- Preoperative preparation:

A-Preparation of the patient:

- Patient consent was taken for spinal and regional anesthesia.

- Maintain the therapeutic drugs (that are not contraindicated with regional anesthesia) till morning of operation.

- Patient informed before the operation about visual analogue scales.

- Peripheral 18 gauge intravenous cannula was inserted and $10 \mathrm{ml} / \mathrm{kg} / \mathrm{hr}$ ringer solution was given as preload.

B- Preparation of the equipment and drugs:

- A standard general anesthesia tray is prepared.

- Standard regional anesthesia tray is prepared with the following equipment:

- Sterile towels and $4 * 4$ gauze packs.

- 25 G Quincke spinal needle.

- Hyperbaric bupivacaine $0.5 \%$.

- Isobaric bupivacaine hydrochloride $0.5 \%$.

- Syringes with local anesthetic lidocaine.

- Sterile gloves, marking pen and surface electrode.

- Fentanyle and midazolam ampule

- Peripheral nerve stimulator.

- Ultrasound machine with a low-frequency $(2-5 \mathrm{MHz})$ curved array probe and a highfrequency $(7-12 \mathrm{MHz})$ linear probe.

- Catheter kit Contiplex®:

${ }^{\circ}$ Including a $10 \mathrm{~cm}$ stimulating needle 18 guage and a catheter 20 guage for CLPB.

${ }^{\circ}$ Including a $4 \mathrm{~cm}$ stimulating needle 18 guage and a catheter 20 guage for CFIB.

- Accufuser@ Varicon silicone balloon infuser control pump.

\section{2- Intraoperative management:}

- Standard monitoring was applied (ECG, NIBP, sPO2) were connected to the patient. Baseline hemodynamic readings were recorded before starting the technique, at the beginning of procedure and every 5 minutes.

- Sedation was achieved with IV midazolam $(0.03 \mathrm{mg} / \mathrm{kg})$ and Fentanyl $(50-75 \mu \mathrm{g})$. 
- Then the patients were assigned to one of two groups according to the block form:

1- Group-1 (CFIB), no=20

2- Group-2 (CLPB), no=20

Patients were divided into two equal groups and were subjected to one of the regional blocks first then spinal anesthesia.

\section{Group-1(CFIB):}

The patient was placed in supine position. The landmarks for this block are the anterior superior iliac spine (ASIS) and the pubic tubercle of the same side. One middle finger was placed on the ASIS and the other middle finger on the pubic tubercle. A line was drawn between these two points. This line was divided into thirds (the index finger of both hands can be used). The point was marked $1 \mathrm{~cm}$ caudal from the junction of the lateral and middle third. This is the injection entry point. With ultrasound (US) the aim is to visually identify the femoral nerve and fascia iliaca and place the local anaesthetic beneath the fascia, lateral to the femoral nerve.

A high frequency ultrasound probe $(13-16 \mathrm{MHz}$, linear array) was used in a transverse direction over the anterior thigh below the inguinal ligament. The needle was advanced until the tip is placed underneath the fascia iliaca and the catheter was introduced.

\section{Group-2 (CLPB):}

The patient was placed in the lateral decubitus position with the side to be operated upper most, and the area was prepared and draped in a sterile fashion. The ultrasound scan was performed using a low frequency, 2-5 MHz, curved array transducer. Liberal amounts of ultrasound gel were applied to the skin over the lumbar paravertebral region for acoustic coupling and the ultrasound transducer was positioned approximately $3-4 \mathrm{~cm}$ lateral and parallel to the lumbar spine, with its orientation marker directed cranially, so as to produce a longitudinal scan of the lumbar paravertebral region.

First, the kidney was visualized; the transducer was then moved caudally, while still maintaining the same orientation, until the sacrum and the L5 transverse process were visible. The lumbar transverse processes were identified by their hyperechoic reflection and an acoustic shadow distal (anterior) to them, which is typical of bone. Once the L5 transverse process was located, the other lumbar transverse processes were identified by counting them from below upwards. The transducer was finally positioned over the L2, L3, and L4 trans- verse processes. Identification of the kidney, peritoneum, and intestine was done first. With the lumbar ultrasound "trident" in view, the lumbar plexus was identified by being hyperechoic structure.

After local anesthetic skin infiltration "lignocaine" $2 \%, 2-3 \mathrm{ml}$, a $10 \mathrm{~cm}, 20$ gauge insulated needle connected to a Peripheral nerve stimulator with initial current intensity of $1.0 \mathrm{~mA}(2 \mathrm{~Hz}, 0.1$ millisecond) was introduced in-plane with the probe. The lumbar plexus was finally identified by eliciting quadriceps contraction at current below $0.4 \mathrm{~mA}$. The catheter was then inserted in the needle and advanced $3 \mathrm{~cm}$ beyond the needle tip. The needle was then withdrawn and the catheter was tunneled and fixed with a sterile medication.

After negative aspiration: Activation of the blocks was performed by injecting $30 \mathrm{ml}$ and $40 \mathrm{ml}$ of $0.125 \%$ bupivacaine in CFIB and CLPB respectively. Injection was done incrementally over 5 minutes. Vital signs monitoring was continued for 30 minutes after bolus dose.

Sensory blockade was assessed in the cutaneous distribution of the femoral nerve (anterior aspect of the thigh), the lateral femoral cutaneous nerve (lateral aspect of the thigh) and the obturator nerve (medial aspect of the thigh) by using cold perception or pinprick. Assessment was done using the 3 point scale for sensory assessment: $0=$ Complete loss, $1=$ Partial loss, $2=$ =Normal sensation.

Motor blockade was assessed by femoral and obturator nerve function (knee extension and thigh adduction). Movement was classified according to modified Bromage scale: No weakness $=0$, partial weakness $=1$, almost complete weakness $=2$, complete weakness $=3$.

All assessements were undertaken approximately every two minutes for 30 minutes. The block was considered unsuccessful if the patient failed to develop decreased sensation over the ipsilateral distal thigh and weakness upon knee extension relative to the contralateral limb within 30 minutes.

After securing the catheter in both groups with tap and benzoin the spinal anesthesia was performed with $3 \mathrm{ml}$ of hyperbaric bupivacaine $0.5 \%(15 \mathrm{mg})$ at L4-L5 interspace with a $25 \mathrm{G}$ Quincke spinal needle after local infiltration of $3 \mathrm{ml}$ of $2 \%$ xylocaine.

At the end of the surgery and after termination of the effect of spinal anaesthesia (3-4 hours), a continuous infusion pump was connected to the 
catheter. Infusion regimen was started by the use of local anesthetic (plain bupivacaine $0.125 \%+$ fentanyle $2 \mathrm{ug} / \mathrm{ml}$ ) at a rate of $6-10 \mathrm{ml} / \mathrm{hr}$ for $48 \mathrm{hrs}$. The catheter was removed after $48 \mathrm{~h}$.

In the surgical ward patients were able to request an additional analgesic. When patients first complained of pain incremental intravenous $2 \mathrm{mg}$ dose of morphine to maintain a resting $\mathrm{VAS}<4$.

\section{1-Postoperative pain assessment by VAS:}

VAS consists of a $10 \mathrm{~cm}$ line with one end labelled "no pain" and the other end labelled "worst pain imaginable". The patient marks the line at the point that best describes the pain intensity. The length of the line to the patient's mark is measured and recorded in millimetres. VAS was assessed at post anaesthesia care unit (PACU) and every 6 hours for 48 hours postoperatively.

2- Time to first require postoperative intravenous morphine and total dose consumption over 48 hours.

\section{3- Patients' Satisfaction:}

The overall level of patient's satisfaction about the procedure and postoperative analgesia was assessed using three-point scale:

$$
\begin{aligned}
& 0=\text { Poor. } \\
& 1=\text { Good } . \\
& 2=\text { Very good } . \\
& 3=\text { Excellent } .
\end{aligned}
$$

Incidence of complications as: (epidural spread, vomiting, nausea and pruiritis) and LA toxicity symptoms and signs were reported.

\section{Statistical methodology:}

Results were presented as mean, SD, absolute numbers and percentage as appropriate. Error bars in the figures represent standard deviation. Statistical analysis of data was done using SPSS (statistical program for social science). Chi-square test was used to compare qualitative variables. Unpaired $t$-test was used to compare two independent groups as regard a quantitative variable. Fisher exact test was used instead of Chi-square test when one expected cell or more $<5$. The level of significance was set at a $p$-value less than 0.05 , while $p$-values $<0.01$ were considered highly significant.

\section{Results}

The study was performed over 40 patients. Five patients were excluded due to difficult catheter insertion or painful needle insertion without heavy sedation. Those patients were substituted with another 5 subjects to allow for completion of sample size.

\section{The patients were divided into two groups:}

Group CFIB $=20$ patients.

Group CLPB $=18$ patients $(2$ patients failed to develop signs of sensory and motor block within 30 minutes of start of activation of the block with failure rate $=10 \%$ ).

\section{Characteristic data:}

The demographic data are presented in (Table 1). No statistically significant difference among both groups.

\section{Postoperative pain assessment:}

In CLPB group, VAS score was significantly better when assessed at PACU $(p<0.001)$. CLPB group requested analgesia significantly later than CFIB group $(p<0.001)$. However, the total consumed dose of morphine over 48 hours postoperative was higher clinically but not statistically in CLPB (Table 2) (Figs.1,2,3).

Sensory and motor assessment over 48 hours postoperative:

Sensory block was more effective in CFIB group but not statistically significant throughout the assessment duration $(p=0.031)$. Motor weakness occurred in CLPB group only ( $p=0.006$ ) (Table 3 ).

Hemodynamic assessment in postoperative period:

Blood pressure values were lower in CLPB group (statistically but not clinically significant) $(p<0.001)$ (Table 4) (Figs. 4,5,6).

Respiratory assessment in postoperative period (Table 5), (Figs. 7,8).

\section{Postoperative VAS score:}

Postoperative VAS scores were significantly higher in CLPB group than in CFIB $(p<0.001)$ (Table 6), (Fig. 9).

\section{Incidence of side effects:}

None of CFIB group patients suffered from epidural spread. On the other hand, 4 patients (22.2\%) of CLPB group experienced epidural spread, representing a significant difference between both groups $(p=0.041)$. Other complications occurred in both groups with no statistically significant difference rates (Table 7).

\section{Patient satisfaction:}

More patients in CFIB group were satisfied than in CLPB ( $p=0.06)$ (Table 8) (Fig. 10). 
Table (1): Patients' characteristics in both study groups.

\begin{tabular}{|c|c|c|c|c|c|}
\hline & $\begin{array}{c}\text { Group=CFIB } \\
\text { group }\end{array}$ & $\begin{array}{c}\text { Group=CLPB } \\
\text { group }\end{array}$ & $t / \chi^{2}$ & df & $p$-value \\
\hline Age (years) & $60.8 \pm 7.1$ & $57.4 \pm 10.4$ & 1.169 & 29.521 & $0.252 \mathbb{I}$ \\
\hline Gender (M/F) & $12 / 8$ & $13 / 5$ & - & & $0.506 \S$ \\
\hline BMI $\left(\mathrm{kg} / \mathrm{m}^{2}\right)$ & $23.8 \pm 3.2$ & $25.2 \pm 3.6$ & -1.290 & 36 & $0.205 \rrbracket$ \\
\hline$A S A-P S$ & & & 0.132 & 1 & $0.717 ¥$ \\
\hline ASA-PS I & $6(30.0 \%)$ & $8(44.4 \%)$ & & & \\
\hline ASA-PS II & $12(60.0 \%)$ & $4(22.2 \%)$ & & & \\
\hline ASA-PS III & $2(10.0 \%)$ & $6(33.3 \%)$ & & & \\
\hline
\end{tabular}

Table (2): Pain score at the PACU, time to first analgesic request, and cumulative morphine consumption in both study groups

\begin{tabular}{|c|c|c|c|c|c|c|c|}
\hline \multirow[t]{2}{*}{$\underline{\text { Variable }}$} & \multicolumn{2}{|c|}{$\begin{array}{l}\text { CFIB group } \\
\quad(\mathrm{n}=20)\end{array}$} & \multicolumn{2}{|c|}{$\begin{array}{l}\text { CLPB group } \\
\quad(n=18)\end{array}$} & \multirow[t]{2}{*}{$t$} & \multirow[t]{2}{*}{ df } & \multirow[t]{2}{*}{$p$-valuedI } \\
\hline & Mean & SD & Mean & SD & & & \\
\hline VAS score for pain at PACU & 2.9 & 1.2 & 1.1 & 0.3 & 6.589 & 22.209 & $<0.001 *$ \\
\hline Time to first analgesic request (h) & 12.4 & 2.0 & 17.2 & 4.9 & -4.023 & 35 & $<0.001 *$ \\
\hline Cumulative 48-h morphine consumption (mg) & 15.4 & 4.4 & 17.2 & 8.0 & -0.854 & 25.775 & 0.401 \\
\hline
\end{tabular}

TIUnpaired $t$-test.

${ }^{*} p$-value $=0.05$ (significant) $<0.001$ (highly significant).

Table (3): Postoperative sensory and motor scores in both study groups.

\begin{tabular}{|c|c|c|c|c|c|c|c|c|c|}
\hline \multirow[t]{2}{*}{ Variable } & \multirow[t]{2}{*}{ Time } & \multicolumn{2}{|c|}{$\begin{array}{l}\text { CFIB group } \\
(\mathrm{n}=20)\end{array}$} & \multicolumn{2}{|c|}{$\begin{array}{l}\text { CLPB group } \\
\quad(\mathrm{n}=18)\end{array}$} & \multirow[t]{2}{*}{75} & \multirow[t]{2}{*}{$\mathrm{U}$} & \multirow[t]{2}{*}{ Z } & \multirow[t]{2}{*}{$p$-valuedI } \\
\hline & & Median & IQR & Median & IQR & & & & \\
\hline \multirow[t]{8}{*}{ Postoperative sensory score } & $6 \mathrm{~h}$ & 1 & $1-2$ & 1 & $1-1$ & 1 & 176.0 & -0.132 & 0.895 \\
\hline & $12 \mathrm{~h}$ & 2 & $1-2$ & 1 & $1-1$ & 1 & 154.0 & -0.864 & 0.388 \\
\hline & $18 \mathrm{~h}$ & 2 & $1-2$ & 1 & $1-1$ & 1 & 120.0 & -2.156 & $0.031 *$ \\
\hline & $24 \mathrm{~h}$ & 2 & $1-2$ & 1 & $1-1$ & 1 & 136.0 & -1.506 & 0.132 \\
\hline & $30 \mathrm{~h}$ & 2 & $1-2$ & 1 & $1-1$ & 1 & 136.0 & -1.506 & 0.132 \\
\hline & $36 \mathrm{~h}$ & 2 & $1-2$ & 1 & $1-1$ & 1 & 136.0 & -1.419 & 0.156 \\
\hline & $42 \mathrm{~h}$ & 2 & $1-2$ & 1 & $1-1$ & 1 & 120.0 & -1.911 & 0.056 \\
\hline & $48 \mathrm{~h}$ & 2 & $1-2$ & 1 & $1-1$ & 1 & 120.0 & -1.911 & 0.056 \\
\hline \multirow[t]{8}{*}{ Postoperative motor score } & $6 \mathrm{~h}$ & 0 & $0-0$ & 0 & $0-0$ & 0 & 160.0 & -1.511 & 0.131 \\
\hline & $12 \mathrm{~h}$ & 0 & $0-0$ & 0 & $0-0$ & 0 & 160.0 & -1.511 & 0.131 \\
\hline & $18 \mathrm{~h}$ & 0 & $0-0$ & 0 & $0-0$ & 0 & 160.0 & -1.511 & 0.131 \\
\hline & $24 \mathrm{~h}$ & 0 & $0-0$ & 0 & $0-0$ & 0 & 160.0 & -1.511 & 0.131 \\
\hline & $30 \mathrm{~h}$ & 0 & $0-0$ & 0 & $0-0$ & 0 & 160.0 & -1.511 & 0.131 \\
\hline & $36 \mathrm{~h}$ & 0 & $0-0$ & 0 & $0-0$ & 0 & 140.0 & -2.199 & $0.028^{*}$ \\
\hline & $42 \mathrm{~h}$ & 0 & $0-0$ & 0 & $0-1$ & 1 & 120.0 & -2.767 & $0.006^{*}$ \\
\hline & $48 \mathrm{~h}$ & 0 & $0-0$ & 0 & $0-1$ & 1 & 120.0 & -2.767 & $0.006^{*}$ \\
\hline
\end{tabular}

IIMann-Whitney test. 
Table (4): Postoperative hemodynamic parameters in both study groups.

\begin{tabular}{|c|c|c|c|c|c|c|c|c|}
\hline \multirow{2}{*}{ Variable } & \multirow{2}{*}{ Time } & \multicolumn{2}{|c|}{ CFIB group $(n=20)$} & \multicolumn{2}{|c|}{ CLPB group $(n=18)$} & \multirow{2}{*}{$t$} & \multirow{2}{*}{ df } & \multirow{2}{*}{$p$-valueII } \\
\hline & & Mean & SD & Mean & SD & & & \\
\hline $\begin{array}{l}\text { Postoperative heart } \\
\text { rate (bpm) }\end{array}$ & $\begin{array}{l}6 \mathrm{~h} \\
12 \mathrm{~h} \\
18 \mathrm{~h} \\
24 \mathrm{~h} \\
30 \mathrm{~h} \\
36 \mathrm{~h} \\
42 \mathrm{~h} \\
48 \mathrm{~h}\end{array}$ & $\begin{array}{l}66.0 \\
68.7 \\
68.8 \\
68.7 \\
70.3 \\
67.9 \\
68.4 \\
68.8\end{array}$ & $\begin{array}{l}6.3 \\
9.0 \\
7.9 \\
8.8 \\
9.6 \\
7.9 \\
8.1 \\
9.3\end{array}$ & $\begin{array}{l}70.1 \\
71.1 \\
70.0 \\
70.8 \\
70.8 \\
72.7 \\
69.9 \\
71.6\end{array}$ & $\begin{array}{l}4.6 \\
4.1 \\
4.7 \\
3.7 \\
4.1 \\
3.6 \\
4.2 \\
5.3\end{array}$ & $\begin{array}{l}-2.283 \\
-1.076 \\
-0.565 \\
-0.926 \\
-0.196 \\
-2.341 \\
-0.696 \\
-1.109\end{array}$ & $\begin{array}{l}36 \\
27.284 \\
36 \\
36 \\
36 \\
36 \\
36 \\
36\end{array}$ & $\begin{array}{l}0.028^{*} \\
0.291 \\
0.576 \\
0.360 \\
0.846 \\
0.025^{*} \\
0.491 \\
0.275\end{array}$ \\
\hline $\begin{array}{l}\text { Postoperative SBP } \\
\text { (mmHg) }\end{array}$ & $\begin{array}{l}6 \mathrm{~h} \\
12 \mathrm{~h} \\
18 \mathrm{~h} \\
24 \mathrm{~h} \\
30 \mathrm{~h} \\
36 \mathrm{~h} \\
42 \mathrm{~h} \\
48 \mathrm{~h}\end{array}$ & $\begin{array}{l}130.0 \\
132.5 \\
133.0 \\
134.5 \\
132.0 \\
131.5 \\
133.5 \\
129.5\end{array}$ & $\begin{array}{l}7.9 \\
7.7 \\
7.3 \\
10.1 \\
6.2 \\
8.3 \\
6.9 \\
5.8\end{array}$ & $\begin{array}{l}111.1 \\
110.0 \\
113.3 \\
111.7 \\
110.8 \\
113.3 \\
112.8 \\
112.8\end{array}$ & $\begin{array}{l}8.7 \\
8.4 \\
7.5 \\
7.1 \\
7.5 \\
7.9 \\
7.7 \\
6.0\end{array}$ & $\begin{array}{l}7.008 \\
8.618 \\
8.183 \\
7.976 \\
9.530 \\
6.913 \\
8.743 \\
8.713\end{array}$ & $\begin{array}{l}36 \\
36 \\
36 \\
36 \\
36 \\
36 \\
36 \\
36\end{array}$ & $\begin{array}{l}<0.001 * \\
<0.001 * \\
<0.001 * \\
<0.001 * \\
<0.001 * \\
<0.001 * \\
<0.001 * \\
<0.001 *\end{array}$ \\
\hline $\begin{array}{l}\text { Postoperative DBP } \\
(\mathrm{mmHg})\end{array}$ & $\begin{array}{l}6 \mathrm{~h} \\
12 \mathrm{~h} \\
18 \mathrm{~h} \\
24 \mathrm{~h} \\
30 \mathrm{~h} \\
36 \mathrm{~h} \\
42 \mathrm{~h} \\
48 \mathrm{~h}\end{array}$ & $\begin{array}{l}78.5 \\
79.5 \\
78.5 \\
77.0 \\
77.0 \\
76.5 \\
76.0 \\
76.0\end{array}$ & $\begin{array}{l}5.6 \\
4.8 \\
5.2 \\
5.7 \\
4.7 \\
4.0 \\
3.8 \\
4.5\end{array}$ & $\begin{array}{l}65.0 \\
66.1 \\
62.2 \\
65.0 \\
65.8 \\
66.4 \\
65.0 \\
66.1\end{array}$ & $\begin{array}{l}4.2 \\
5.3 \\
6.5 \\
6.4 \\
3.5 \\
5.4 \\
3.4 \\
6.3\end{array}$ & $\begin{array}{l}8.287 \\
8.140 \\
8.620 \\
6.100 \\
8.200 \\
6.621 \\
9.272 \\
5.615\end{array}$ & $\begin{array}{l}36 \\
36 \\
36 \\
36 \\
36 \\
36 \\
36 \\
36\end{array}$ & $\begin{array}{l}<0.001 * \\
<0.001 * \\
<0.001 * \\
<0.001 * \\
<0.001 * \\
<0.001 * \\
<0.001 * \\
<0.001 *\end{array}$ \\
\hline
\end{tabular}

IIUnpaired $t$-test.

Table (5): Postoperative respiratory parameters in both study groups.

\begin{tabular}{|c|c|c|c|c|c|c|c|c|}
\hline \multirow{2}{*}{ Variable } & \multirow{2}{*}{ Time } & \multicolumn{2}{|c|}{ CFIB group $(n=20)$} & \multicolumn{2}{|c|}{ CLPB group $(n=18)$} & \multirow{2}{*}{$t$} & \multirow{2}{*}{ df } & \multirow{2}{*}{$p$-valueII } \\
\hline & & Mean & SD & Mean & SD & & & \\
\hline \multirow{8}{*}{$\begin{array}{l}\text { Postoperative } \\
\text { RR (bpm) }\end{array}$} & $6 \mathrm{~h}$ & 16.2 & 1.3 & 15.1 & 1.2 & 2.664 & 36 & $0.011 *$ \\
\hline & $12 \mathrm{~h}$ & 17.3 & 3.8 & 15.1 & 1.6 & 2.313 & 36 & $0.027 *$ \\
\hline & $18 \mathrm{~h}$ & 16.2 & 1.2 & 15.7 & 1.7 & 1.020 & 36 & 0.314 \\
\hline & $24 \mathrm{~h}$ & 16.5 & 1.1 & 15.8 & 1.1 & 2.008 & 36 & 0.052 \\
\hline & $30 \mathrm{~h}$ & 15.9 & 1.0 & 15.7 & 1.5 & 0.587 & 36 & 0.561 \\
\hline & $36 \mathrm{~h}$ & 16.5 & 0.7 & 15.5 & 1.8 & 2.191 & 21.327 & $0.040^{*}$ \\
\hline & $42 \mathrm{~h}$ & 16.8 & 0.6 & 16.2 & 1.4 & 1.681 & 36 & 0.102 \\
\hline & $48 \mathrm{~h}$ & 15.2 & 1.0 & 16.0 & 2.1 & -1.547 & 36 & 0.131 \\
\hline \multirow{8}{*}{$\begin{array}{c}\text { Postoperative } \\
\mathrm{SpO}_{2}(\%)\end{array}$} & $6 \mathrm{~h}$ & 99.6 & 0.5 & 99.4 & 0.5 & 0.945 & 36 & 0.351 \\
\hline & $12 \mathrm{~h}$ & 99.8 & 0.4 & 100.0 & 0.0 & -2.179 & 19.000 & $0.042 *$ \\
\hline & $18 \mathrm{~h}$ & 99.8 & 0.4 & 100.0 & 0.0 & -2.179 & 19.000 & $0.042 *$ \\
\hline & $24 \mathrm{~h}$ & 99.2 & 0.4 & 100.0 & 0.0 & -8.718 & 19.000 & $<0.001 *$ \\
\hline & $30 \mathrm{~h}$ & 99.2 & 0.4 & 100.0 & 0.0 & -8.718 & 19.000 & $<0.001 *$ \\
\hline & $36 \mathrm{~h}$ & 99.2 & 0.4 & 99.0 & 0.0 & 2.179 & 19.000 & $0.042 *$ \\
\hline & $42 \mathrm{~h}$ & 99.8 & 0.4 & 99.0 & 0.0 & 8.718 & 19.000 & $<0.001 *$ \\
\hline & $48 \mathrm{~h}$ & 99.8 & 0.4 & 99.0 & 0.0 & 8.718 & 19.000 & $<0.001 *$ \\
\hline
\end{tabular}

IUUnpaired $t$-test. $* p$-value $=0.05$ (significant) $<0.001$ (highly significant).

Table (6): Postoperative VAS score for pain in both study groups.

\begin{tabular}{|c|c|c|c|c|c|c|c|c|}
\hline \multirow{2}{*}{ Variable } & \multirow{2}{*}{ Time } & \multicolumn{2}{|c|}{ CFIB group $(n=20)$} & \multicolumn{2}{|c|}{ CLPB group $(n=18)$} & \multirow{2}{*}{$t$} & \multirow{2}{*}{ df } & \multirow{2}{*}{$p$-valued } \\
\hline & & Mean & SD & Mean & $\mathrm{SD}$ & & & \\
\hline \multirow{8}{*}{$\begin{array}{l}\text { Postoperative VAS } \\
\text { score for pain }\end{array}$} & $6 \mathrm{~h}$ & 1.7 & 0.9 & 1.6 & 0.5 & 0.363 & 36 & 0.719 \\
\hline & $12 \mathrm{~h}$ & 1.9 & 1.2 & 1.9 & 0.7 & -0.139 & 36 & 0.890 \\
\hline & $18 \mathrm{~h}$ & 1.6 & 0.7 & 2.3 & 0.8 & -2.917 & 36 & $0.006^{*}$ \\
\hline & $24 \mathrm{~h}$ & 2.0 & 1.5 & 2.6 & 0.9 & -1.366 & 36 & 0.181 \\
\hline & $30 \mathrm{~h}$ & 2.0 & 0.9 & 2.9 & 1.3 & -2.496 & 29.524 & $0.018 *$ \\
\hline & $36 \mathrm{~h}$ & 2.2 & 0.9 & 3.7 & 1.5 & -3.550 & 26.754 & $0.001 *$ \\
\hline & $42 \mathrm{~h}$ & 1.9 & 1.0 & 4.4 & 1.8 & -5.256 & 25.569 & $<0.001 *$ \\
\hline & $48 \mathrm{~h}$ & 2.4 & 0.7 & 5.1 & 2.2 & -5.031 & 19.940 & $<0.001 *$ \\
\hline
\end{tabular}

qUnpaired $t$-test. $* * p$-value $=0.05$ ( significant $)<0.001$ (highly significant). 
Table (7): Incidence of unwanted outcomes in both study groups.

\begin{tabular}{lccc}
\hline Variable & Group=CFIB group & Group=CLPB group & $p$-value \\
\hline Epidural spread & $0(0.0 \%)$ & $4(22.2 \%)$ & $0.041^{*}$ \\
Nausea & $2(10.0 \%)$ & $6(33.3 \%)$ & 0.117 \\
Vomiting & $2(10.0 \%)$ & $6(33.3 \%)$ & 0.117 \\
Pruritus & $2(10.0 \%)$ & $6(33.3 \%)$ & 0.117 \\
\hline
\end{tabular}

Data are number $(\%)$

Fisher's exact test.

${ }^{*} p$-value $=0.05$ (significant $)<0.001$ (highly significant).

Table (8): Patient satisfaction score in both study groups.

\begin{tabular}{|c|c|c|c|c|c|c|c|}
\hline \multirow{2}{*}{ Variable } & \multicolumn{2}{|c|}{$\begin{array}{l}\text { CFIB group } \\
(\mathrm{n}=20)\end{array}$} & \multicolumn{3}{|c|}{$\begin{array}{l}\text { CLPB group } \\
\quad(\mathrm{n}=18)\end{array}$} & \multirow{2}{*}{$\mathrm{Z}$} & \multirow{2}{*}{$p$-value } \\
\hline & Median & IQR & Mean & Median & IQR & & \\
\hline Patient satisfaction score & 9.0 & $8.0-9.0$ & 8.0 & $7.0-9.0$ & 120.0 & -1.879 & $0.060^{*}$ \\
\hline
\end{tabular}

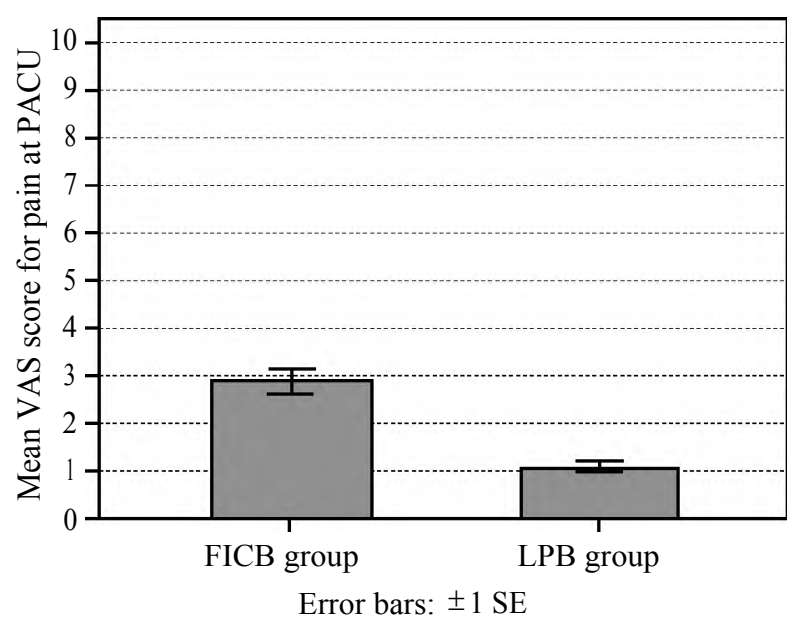

Fig. (1): Mean VAS score for pain at the PACU in both study groups. Error bars represent the standard error of the mean (SEM).

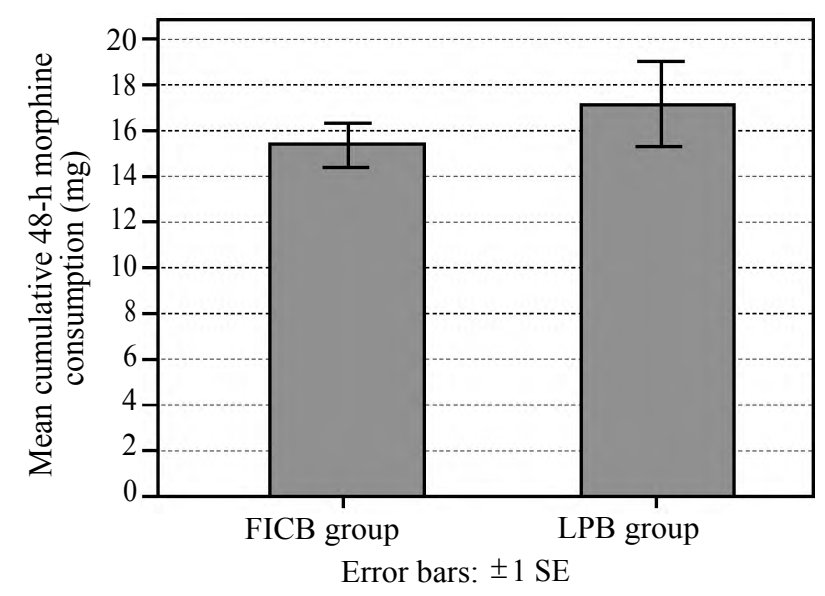

Fig. (3): Mean cumulative 48-h morphine consumption in both study groups.

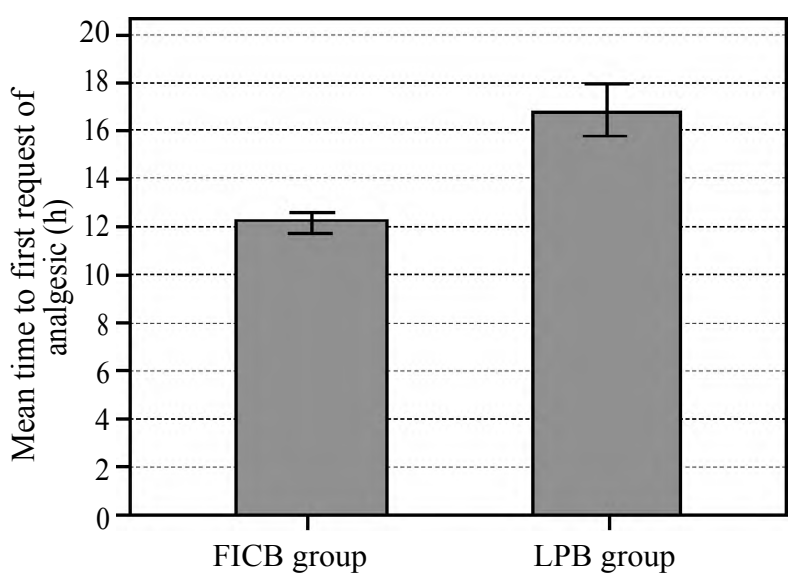

Error bars: $\pm 1 \mathrm{SE}$

Fig. (2): Mean time to first analgesic request in both study groups. Error bars represent the standard error of the mean (SEM).

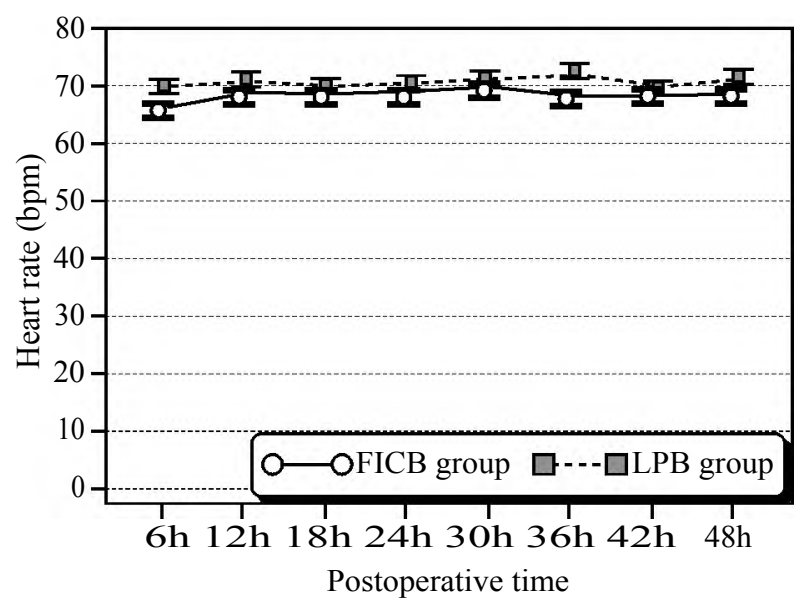

Fig. (4): Mean postoperative heart rate in both study groups. Error bars represent the standard error of the mean (SEM). 

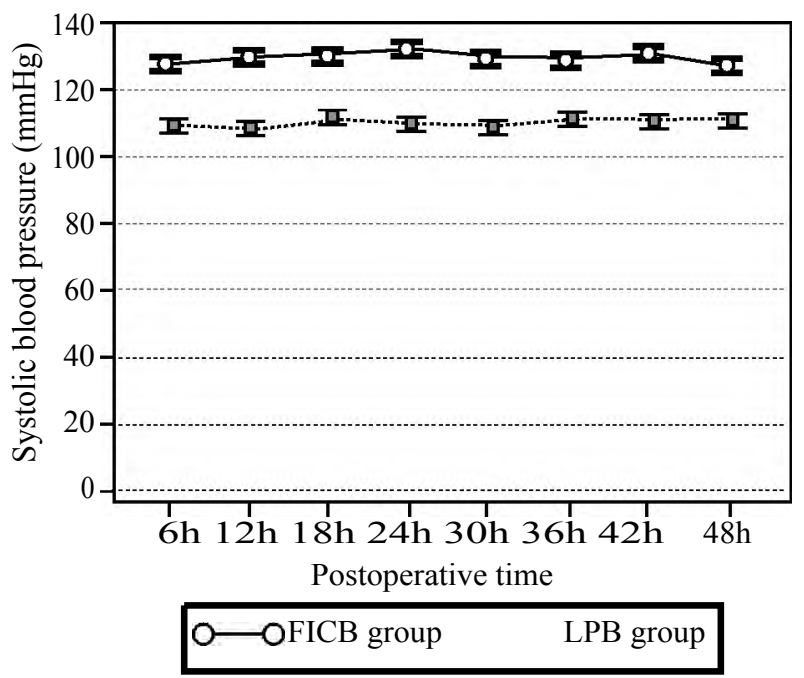

Fig. (5): Mean postoperative systolic blood pressure in both study groups. Error bars represent the standard error of the mean (SEM).

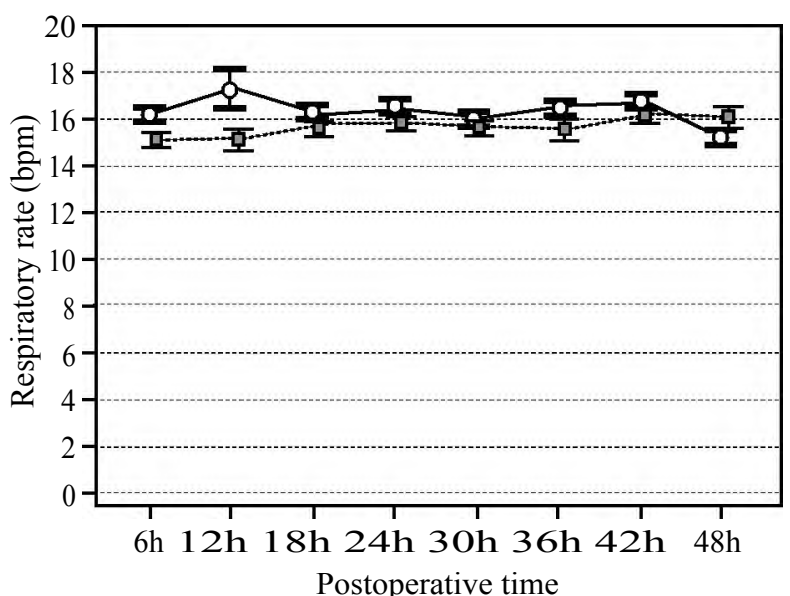

O-OFICB group LPB group

Fig. (7): Mean postoperative respiratory rate in both study groups. Error bars represent the standard error of the mean (SEM).

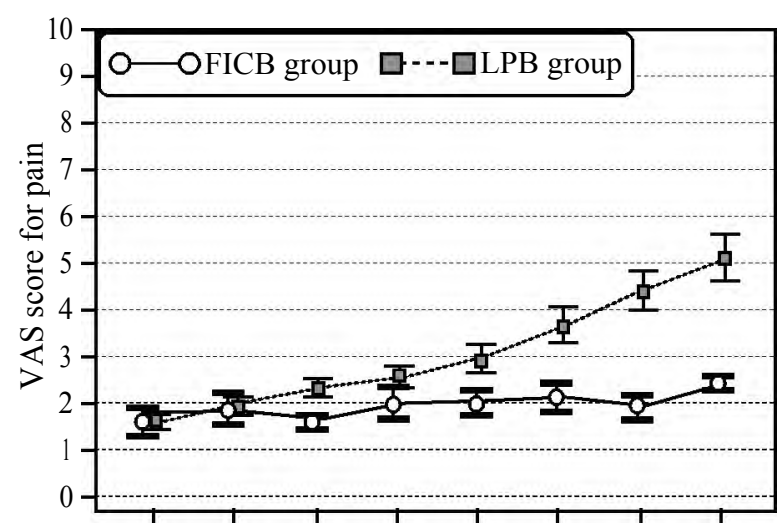

6h 12 h 18 h 24 h $30 h 36 h 42$ h 48 h Postoperative time

Fig. (9): Mean postoperative VAS score for pain in both study groups. Error bars represent the standard error of the mean (SEM).

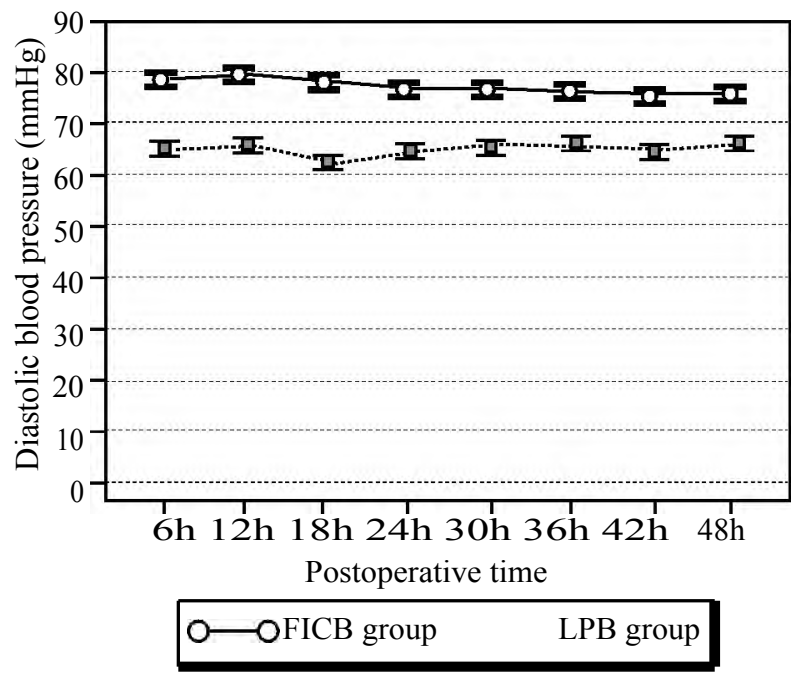

Fig. (6): Mean postoperative diastolic blood pressure in both study groups. Error bars represent the standard error of the mean (SEM)

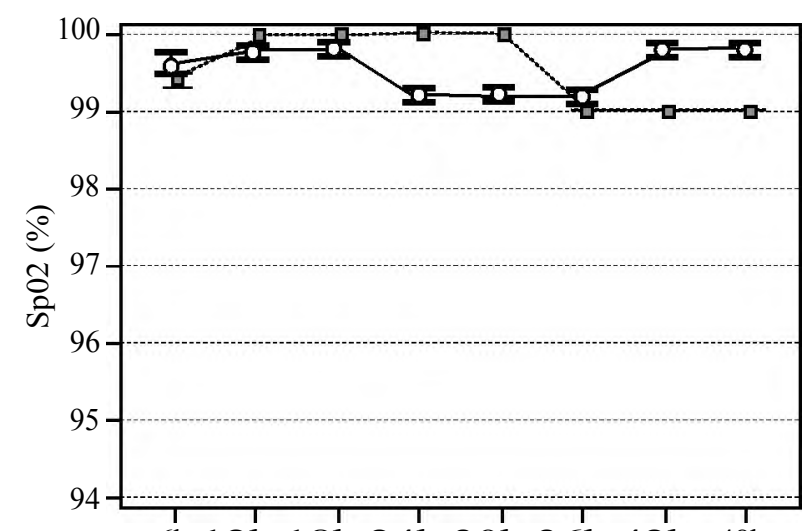

6h $12 \mathrm{~h} 18 \mathrm{~h} 24 \mathrm{~h} 30 \mathrm{~h} 36 \mathrm{~h} 42 \mathrm{~h} 48 \mathrm{~h}$

Postoperative time

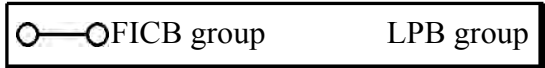

Fig. (8): Mean postoperative $\mathrm{SpO} 2$ in both study groups. Error bars represent the standard error of the mean (SEM).

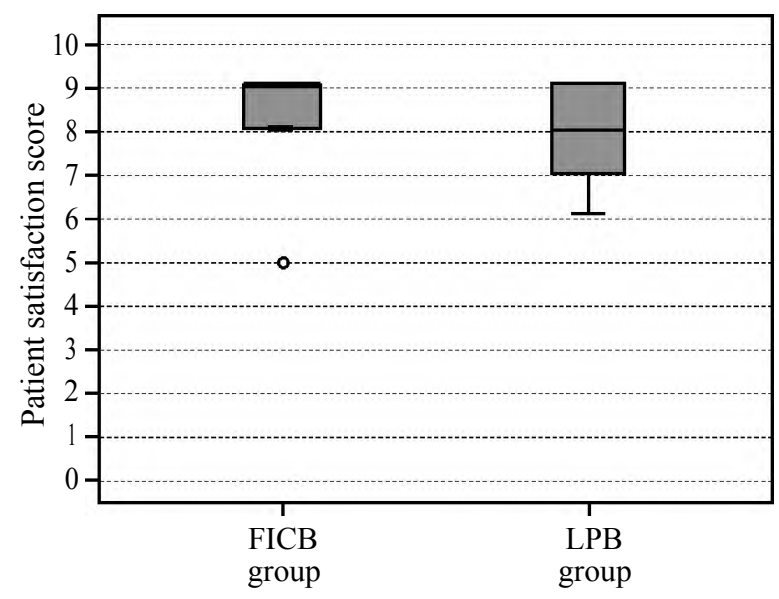

Fig. (10): Box plot showing the patient satisfaction score in both study groups. 


\section{Discussion}

Hip fractures are painful and pain left untreated can result in complications that may delay operative intervention and complicate hospital stay [5]. Pain management in many hospitals is based upon the use of systemic analgesia. Many guidelines suggest considering the use of neural blockade by trained personnel to limit opioid dosage and its complications [6].

The present study compares postoperative analgesia in continuous facia iliaca block (CFIB) and continuous lumbar plexus block (CLPB) in patients with hip fractures.

When studying VAS scores in post anaesthesia care unit (PACU) in the current study they were significantly lower in CLPB group (mean $=2.9 \pm 1.2$ ) than in CFIB (mean $=1.1 \pm 0.3)(p<0.001)$. Jacques et al., said that LPB resulted in statistically significant reductions in PACU resting pain, compared to multimodal analgesia (neuraxial anesthesia, ketorolac, hydrocodone/acetaminophen, and IV hydromorphone) [7]. Biboulet et al., also studied the analgesic potency of a single injection LPB over PCA with intravenous morphine and a femoral nerve block. He found that the total morphine consumption was significantly lower in LPB group $(p=0.002)[8]$

In the present study, sensory and motor assessment within 48 hours postoperative in CFIB group showed statistically significant higher efficacy of sensory block when compared to CLPB $(p=0.031)$. While motor power wasn't affected in CFIB as the dose of infusion was sensory dose. Despite the fact that the infusion dose in CLPB group was also a sensory dose, motor weakness occurred probably due to quadriceps femoris weakness. In their study, Nie et al., concluded that the CFIB technique provided longer analgesia than a single FIB and is suitable for postoperative analgesia [9]. Although the obturator nerve has been reported to be blocked better by the LPB bolus dose, the block started to decrease postoperative. This can be explained by the lower concentration and volume of the local anaesthetic given as infusion than loading dose [10]. Quadriceps femoris weakness is a possible disadvantage associated with LPB with added functional disability. Early physiotherapy would decrease motor weakness [11]. CLPB in old patients undergoing major lower extremity orthopaedic surgery is associated with an increased risk for postoperative falls compared with non-continuous blockade or no blockade [12]. Several previous studies discussed the relation between infusion rate and degree of quadriceps femoris weakness. The motor weakness was found to be avoidable by minimizing the local anaesthetic dose in perineural infusion. It was found that among patients receiving ropivacaine $0.2 \% 8 \mathrm{~mL} / \mathrm{hr}, 43 \%$ experienced quadriceps femoris weakness which resulted in an increase risk of falls [13]. Ilfeld et al., recommended that if patients receiving ropivacaine $0.2 \%$ at $6 \mathrm{~mL} / \mathrm{hr}$ experienced quadriceps femoris weakness, the basal administration rate should be reduced by half (to $3 \mathrm{~mL} / \mathrm{hr}$ ) [14]. A more recent study considered a ropivacaine $0.2 \%$ rate of $4 \mathrm{~mL} / \mathrm{hr}$ to be appropriate in minimizing the risk of falling after THA [15]. In their study, Sayed and Yousef, found that the cocentration of $0.125 \%$ levobupivacaine at a rate of $5 \mathrm{ml} / \mathrm{h}$ produced sensory rather than motor block effectively with no falling episodes among study patients [11]. A reduction in opoid usage during hospitalization for hip fractures reduces the risk of opoid induced side effects: vomiting, nausea, pruiritis, acute delirium, urine retention and constipation [16]

Regarding morphine request, CFIB group required morphine earlier (mean $=12.4 \pm 2$ hours) than LPB group (mean $=17.2 \pm 4.9$ hours) $(p<0.001)$. The total consumed dose of morphine over 48 hours postoperative was only slightly higher in CLPB group (mean $=17.2 \pm 8 \mathrm{mg}$ ) than CFIB (mean $=15.4 \pm$ $4.4 \mathrm{mg})(\mathrm{p}=0.401)$. This is consistent with Srivastava et al, who described longer duration of analgesia in CLPB group, mean time of 1 st demand of morphine $12.4 \pm 7.9$ hours postoperatively, compared with CFICB group (mean=10.7 \pm 6.4$)$ [17]. In their study, Dauri et al., suggested that the lower overall opioid consumption in patients with CLPB is mostly attributable to higher rate of obturator nerve territory involvement compared to other blocks [18] This was also described by Marino et al, who found that both CLPB and femoral block significantly reduce postoperative opioid requirements [19] Turker et al., in a study to compare between LPB and epidural analgesia for patients undergoing hip surgery, described no significant difference in analgesic potency [20]. This implies a certain preference for LPB as a postoperative analgesia strategy for hip surgery because undesired side effects of epidural analgesia such as urine retention, hypotension, respiratory depression and epidural hematoma are avoided and the possibility of prolonged postoperative analgesia can be maintained [21]. Marino et al., described that patients with CLPB walked significantly greater distances than those in either the continuous femoral block or patients controlled analgesia groups. The facts that CLPB provides improved postoperative analgesia, 
less motor block, and lower prevalence of opioid related side effects are the most likely causes [19]

In the current study, postoperative VAS scores were significantly lower in CFIB group than in CLPB $(p<0.001)$. Stevens et al., compared patients receiving single injection $L P B$ combined with general anaesthesia and patients who did not receive LPB. They described significantly lower VAS ( $p=$ $0.007)$ and cumulative postoperative morphine consumption $(p<0.001)$ in LPB group [22] . Becchi et al., described lower pain scores and less needed rescue analgesia over 48 hours postoperative after total hip arthroplasty in patients with CLPB [23]

Studying patient satisfaction, in the present study, results of patient satisfaction for both groups were high, with non-significant preference in favour of CFIB ( 9 out of 10) in comparison with CLPB ( 8 out of 10$)(p=0.06)$. In 2007, Srivastata et al., compared FIB and LPB in patients for hip surgery. They found that more than $90 \%$ of patients in both groups graded their analgesia as excellent or good [17]. This was also proved by Seddiqui et al., who concluded that CLPB reduced opioid requirements and enhanced patient satisfaction compared with systemic opioids following hip replacement [24] This is in agreement with Abdelmawgoud and Rashwan, who concluded that both CLPB and CFIB provided good quality of analgesia and patient satisfaction during 1 st 24 hours postoperative after hip surgery as evidenced by low VAS and low postoperative analgesic requirements [25]

Assessment of incidence of side effects in both groups showed that none of CFIB group suffered from epidural spread. On the other hand, 4 patients of CLPB group experienced epidural spread ( $p=$ 0.04 ). Nausea, vomiting and pruiritis occurred in both groups with higher frequency in CLPB, but not statistically significant $(p=0.117)$. Other complications as local anaesthetic toxicity, retroperitoneal hematoma and total spinal anaesthesia were not reported in CLPB. Dolan et al., said that, the most frequently occuring side effects in LPB is the epidural diffusion of the injected local anesthetic. Reported incidence vary between 3-27\% [26]. A medial needle insertion point and a more cephalid lumbar approach L2-L3 of the LPB seems to be prognostic risk factors for this undesirable side effect [2]. However, Mannion described that a large injected volume is probably the most important prognostic factor for bilateral spread and not the approach of LPB [27]. Another important factor which could influence the occurrence of epidural diffusion of local anesthetic after LPB is the pressure during injection [21]. Gadsden et al., concluded that injection of local anaesthetic with high injection pressure (>20 psi) during LPB commonly results in unwanted bilateral blockade and is associated with high risk of neuroaxial blockade [28]. The use of combined technique involving ultrasound guided approach and peripheral nerve stimulator in LPB helps in the reduction of complications as LA systemic toxicity, total spinal block, renal injury and retroperitoneal hematoma and improved safety profile [11].

A new emerging Sharmock's technique may carry hope in avoidance of complications linked to other known techniques. Scanning from the patient's flank in a transverse plane gives an improved view of the lumbar plexus with better identification of the anatomical structures surrounding the lumbar plexus. This is facilitated by the well recognisable ultrasonographic pattern of a shamrock with three leaves. By advancing the needle in-plane and perpendicular to the ultrasound beam, the needle tip can be visualised clearly and positioned precisely beside the target nerves. However, data are still missing limiting its use [29]

In their study, Nie et al., reported less frequency of post-operative nausea, vomiting and pruiritis among FICB group compared to PCA. This was consistent with the lower dose of opioids received by FIB group [9]. The use of ultrasound guidance for FIB significantly improves the incidence motor and sensory block [26]. Thus, helps lowering the opioid dose more and more with consequent decreased risk of side effects

\section{Conclusions:}

Single injection lumbar plexus block provides postoperative analgesia for up to 18 hours, with avoidance of troubles of continuous infusion, with lower VAS scores and good patient satisfaction. However, it is considered a difficult technique with higher rate of complications and failure. On the other hand, Continuous infusion fascia iliaca block gives better quality analgesia, for up to 48 hours, and high patient satisfaction.

\section{References}

1- RANGE C. and EGELER C.: Fascia iliaca compartment block: landmark and ultrasound approach. Anaesthetic Tutorial of the Week, 193: 1-4, 2010.

2- HILLER D.B., di GREGORIO G., KELLY K., RIPPER R, EDELMAN L., BOUMENDJEL R., DRASNER K. and WEINBERG G.L.: Safety of high volume lipid emulsion infusion: A first approximation of LD 50 in rats. Reg. Anesth. Pain Med., 35: 140-4, 2010. 
3- DULANEY-CRIPE E., HADAWAY S., BAUMAN R., TRAME C., SMITH C., SILLAMAN B. and LAUGHLIN R.: A continuous infusion fascia iliaca compartment block in hip fracture patients: a pilot study. Journal of Clinical Medicine Research Feb., 4 (1): 45, 2012.

4- MARHOFER P., WILLSCHKE H., GREHER M. and KAPRAL S.: New perspectives in regional anesthesia: the use of ultrasound-past, present, and future. Canadian Journal of Anesthesia/Journal canadien d'anesthésie. Jun., 1, 52: R28-32, 2005

5- VISCUSI E.R. and PAPPAGALLO M.: A review of opioids for in-hospital pain management. Hospital Practice. Feb., 1, 40 (1): 149-59, 2012.

6- HANNA L., GULATI A. and GRAHAM A.: The Role of Fascia Iliaca Blocks in Hip Fractures: A Prospective CaseControl Study and Feasibility Assessment of a JuniorDoctor-Delivered Service. ISRN Orthopedics. Mar., 4, 2014.

7- YADEAU J.T., TEDORE T., GOYTIZOLO E.A., KIM D.H., GREEN D.S., WESTRICK A., FAN R., RADE M.C., RANAWAT A.S., COLEMAN S.H. and KELLY B.T.: Lumbar plexus blockade reduces pain after hip arthroscopy: A prospective randomized controlled trial. Anesthesia \& Analgesia. Oct., 1, 115 (4): 968-72, 2012.

8- BIBOULET P., MORAU D., AUBAS P., BRINGUIERBRANCHEREAU S. and CAPDEVILA X.: Postoperative analgesia after total-hip arthroplasty: Comparison of intravenous patient-controlled analgesia with morphine and single injection of femoral nerve or psoas compartment block. A prospective, randomized, double-blind study. Regional anesthesia and pain medicine. Apr., 30, 29 (2): 102-9, 2004.

9- NIE H., YANG Y.X., WANG Y., LIU Y., ZHAO B. and LUAN B.: Effects of continuous fascia iliaca compartment blocks for postoperative analgesia in patients with hip fracture. Pain Research and Management, 20 (4): 210-2, 2015.

10- ÖZALP G., KAYA M., TUNCEL G., CANOLER Ö., GÜLNERMAN G., ŞAVL1 S. and KADıOGULLARı N.: The analgesic efficacy of two different approaches to the lumbar plexus for patient-controlled analgesia after total knee replacement. Journal of Anesthesia. Aug., 1, 21 (3): 409-12, 2007.

11-SAYED A.M. and YOUSEF K.: Continuous femoral nerve against psoas compartment block for analgesia in total knee arthroplasty. Ain-Shams Journal of Anaesthesiology. Apr 1; 8 (2): 200, 2015.

12- JOHNSON R.L., KOPP S.L., HEBL J.R., ERWIN P.J. and MANTILLA C.B.: Falls and major orthopaedic surgery with peripheral nerve blockade: A systematic review and meta-analysis. British Journal of Anaesthesia. Apr., 1, 110 (4): 518-28, 2013.

13- ILFELD B.M., LE L.T., RAMJOHN J., LOLAND V.J., WADHWA A.N., GERANCHER J.C., RENEHAN E.M., SESSLER D.I., SHUSTER J.J., THERIAQUE D.W. and MALDONADO R.C.: The effects of local anesthetic concentration and dose on continuous infraclavicular nerve blocks: a multicenter, randomized, observer-masked, controlled study. Anesthesia and Analgesia. Jan., 108 (1): $345,2009$.
14- ILFELD B.M., MARIANO E.R., MADISON S.J., LOLAND V.J., SANDHU N.S., SURESH P.J, BISHOP ML, KIM T.E., DONOHUE M.C., KULIDJIAN A.A. and BALL S.T.: Continuous femoral versus posterior lumbar plexus nerve blocks for analgesia after hip arthroplasty: a randomized, controlled study. Anesthesia and Analgesia. Oct., 113 (4): 897, 2011.

15- TETSUNAGA T., SATO T., SHIOTA N., TETSUNAGA T., YOSHIDA M., OKAZAKI Y. and YAMADA K.: Comparison of continuous epidural analgesia, patient-controlled analgesia with morphine, and continuous three-in-one femoral nerve block on postoperative outcomes after total hip arthroplasty. Clinics in Orthopedic Surgery. Jun., 1, 7 (2): 164-70, 2015.

16- KANDASAMI M., KINNINMONTH A.W., SARUNGI M., BAINES J. and SCOTT N.B.: Femoral nerve block for total knee replacement: A word of caution. Knee, 16 (2): 98-100, 2009.

17- SRIVASTAVA U., KUMAR A., SAXENA S., NAZ A., GOYAL V. and MEHROTRA R.: Lumbar plexus block for post-operative analgesia following hip surgery: A comparison of " 3 in 1" and psoas compartment block. Indian Journal of Anaesthesia. Mar., 1, 51 (2): 127, 2007.

18- DAURI M., FARIA S., CELIDONIO L., DAVID P., BIANCO A., FABBI E. and SILVI MB.: The Comparing of Ultrasound-guided Techniques: Sciatic Block with Continuous Lumbar Plexus Block or Continuous Femoral Nerve Block for Anesthesia and Analgesia of Total knee Replacement. Open Anesthesiology Journal, 7: 19-25, 2013.

19- MARINO J., RUSSO J., KENNY M., HERENSTEIN R., LIVOTE E. and CHELLY J.E.: Continuous lumbar plexus block for postoperative pain control after total hip arthroplasty. The Journal of Bone \& Joint Surgery. Jan., 1, 91 (1): 29-37, 2009.

20- TÜRKER G., UCKUNKAYA N., YAVAŞÇAOGLU B. YILMAZLAR A. and ÖZÇELIK S.: Comparison of the catheter-technique psoas compartment block and the epidural block for analgesia in partial hip replacement surgery. Acta anaesthesiologica scandinavica. Jan., 1, 47 (1): 30-6, 2003.

21- DE LEEUW M.A., ZUURMOND W.W. and PEREZ R.S. The psoas compartment block for hip surgery: The past, present, and future. Anesthesiology Research and Practice. May., 22, 2011.

22- STEVENS R.D., VAN GESSEL E., FLORY N., FOURNIER R. and GAMULIN Z.: Lumbar plexus block reduces pain and blood loss associated with total hip arthroplasty. The Journal of the American Society of Anesthesiologists. Jul., 1, 93 (1): 115-21, 2000.

23- BECCHI C., AL MALYAN M., COPPINI R., CAMPOLO M., MAGHERINI M. and BONCINELLI S.: Opioid-free analgesia by continuous psoas compartment block after total hip arthroplasty. A randomized study. Eur. J. Anaesthesiol., 25: 418-423, 2008.

24- SIDDIQUI Z.I., CEPEDA M.S., DENMAN W., SCHU MANN R. and CARR D.B.: Continuous lumbar plexus block provides improved analgesia with fewer side effects compared with systemic opioids after hip arthroplasty: A randomized controlled trial. Regional anesthesia and pain medicine. Sep., 30, 32 (5): 393-8, 2007. 
25- ABDELMAWGOUD A. and RASHWAN S.: The analgesic efficacy of continuous fascia iliaca block vs. continuous psoas compartment block after hip surgery: A comparative study. Egyptian Journal of Anaesthesia. Jul., 31, 28 (3): 183-7, 2012.

26- DOLAN J., WILLIAMS A., MURNEY E., SMITH M. and KENNY G.N.: Ultrasound guided fascia iliaca block: A comparison with the loss of resistance technique. Regional Anesthesia and Pain Medicine. Dec., 31, 33 (6): 526-31, 2008

27- MANNION S.: Psoas compartment block. Continuing
Education in Anaesthesia, Critical Care \& Pain. Oct., 1, 7 (5): 162-6, 2007.

28- GADSDEN J., McCALLY C. and HADZIC A.: Monitoring during peripheral nerve blockade. Current Opinion in Anesthesiology. Oct., 1, 23 (5): 656-61, 2010.

29- SAUTER A.R., ULLENSVANG K., NIEMI G., LORENTZEN H.T., BENDTSEN T.F., BØRGLUM J., PRIPP A.H. and ROMUNDSTAD L.: The Shamrock lumbar plexus block The minimum effective local anaesthetic volume for a new ultrasound-guided lumbar plexus technique. Eur. J. Anaesthesiol., 32: 1-7, 2015.

\title{
مستوى رضا المرضى بعد الجراحة فى حالات كسر عظمة الفخذ الفي

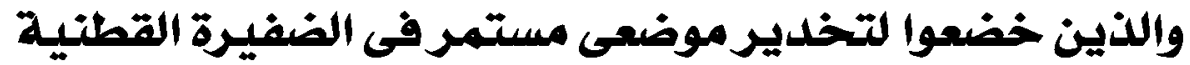

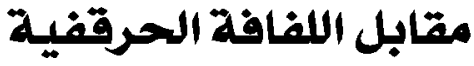

\author{
الخلفيدة: من المعتقد أن كلاً من CLPB, CFIB يمكن أن يقلل من مدة الإقامة فى المستشفى والأعتلال، ويساعد على التمشى المبكر

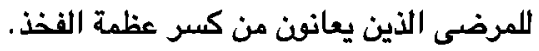 \\ الهدف من الدراسة: لمقارنة فاعلية تسكين الألم وتآثير تقليل تعاطى المهدئات ما بعد الجراحة في المرضى الذين يخضعقن لعملية جراحية

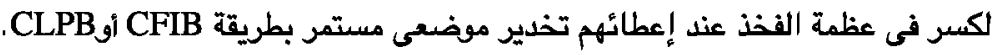 \\ المواد و الطرق: تم عشوائية . ع مريضاً يعانف من كسر فى عظمة الفخذ إلى CLPB, CFIB. \\ النتائج: كان CFIB متفوقة على CLPB في أجمالى الجرعة المستهلكة من الموفين وكتلة الصسية و الصركية لمدة ^ع ساعة بعد العمل

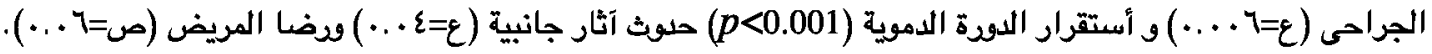

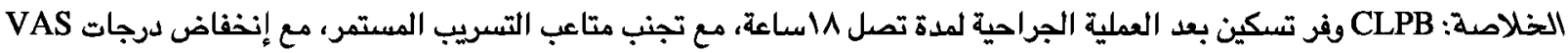

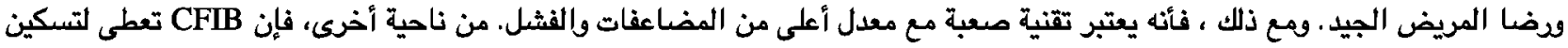

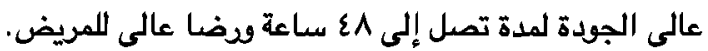

